\title{
LÍQUENES EPíFITOS DE ZONAS ÁRIDAS. EL SABINAR DE LA RETUERTA DE PINA (LOS MONEGROS, ESPAÑA)
}

\author{
Javier ETAYO y Javier BLASCO ZUMETA
}

\begin{abstract}
RESUMEN. Se señalan un total de 66 táxones, entre líquenes y hongos liquenícolas, que colonizan las comunidades arbustivas y arbóreas del sabinar de la Retuerta de Pina, enclavada en Los Monegros (Zaragoza), una de las zonas más áridas de Europa. Pyrenochaeta xanthoriae, Trichothecium roseum y Thelenella justii son nuevas citas peninsulares. Otros táxones están en estudio, dados sus rasgos diferenciales con especies conocidas. Son los siguientes: Agonimia cf. octospora, Bacidia hegetschweileri, Caloplaca cf. viperiae., Rinodina cf. physciospora y Sclerophora sp.
\end{abstract}

Palabras clave. Líquenes, epifitos, sabinar, Los Monegros, España.

ABSTRACT. 66 taxa of epiphytic lichens and lichenicolous fungi are recorded from La Retuerta de Pina, Zaragoza, Spain. This region is one of the most arid from Europe. Pyrenochaeta xanthoriae, Trichothecium roseum and Thelenella justii are recorded the first time in the Peninsula. Other taxa too: Agonimia cf. octospora, Bacidia hegetschweileri, Caloplaca cf. viperiae., Rinodina cf. physciospora and Sclerophora sp. remain under study.

Key words. Lichens, epiphytes, Juniperus thurifera wood, Los Monegros, Spain.

\section{INTRODUCCIÓN}

La comarca de Los Monegros es una de las regiones más desertizadas de la Península Ibérica. Enclavada en el centro de la Depresión Terciaria del Valle Medio del Ebro (fig. 1), está sometida a unas condiciones climáticas extremas, que han configurado un tapiz vegetal propio de las estepas mauritánicas (Braun-Blanquet y Bolós, 1957).

Efectivamente, la pantalla formada por los Pirineos, Sistema Ibérico y Cordillera Litoral y Prelitoral Catalana, cierra la zona a las influencias, tanto del Cantábrico, como del Mediterráneo, definiendo así un clima continental árido, caracterizado por los siguientes parámetros (Ochoa, 1982): Gran oscilación entre las temperaturas extremas anuales que varían desde $-10^{\circ} \mathrm{C}$ hasta superar los $40^{\circ} \mathrm{C}$. Frecuentes heladas primaverales. Inversión térmica muy acusada en invierno, que provoca mínimas que no son propias de esta latitud $\left(41^{\circ} 39^{\prime}\right)$. Este fenómeno se acentúa con la repartición local de nieblas persistentes en los fondos más bajos (generalmente por debajo de los $400 \mathrm{~m}$ ), mientras la atmósfera permanece despejada por encima de los mismos. 
Vientos dominantes del NO y SE (viento frío y cálido respectivamente) con gran capacidad desecadora e importante aumento de la evapotranspiración. Pluviometría media anual escasa (200-400 mm) con máximos primaverales y otoñales. Déficit hídrico superior a los $300 \mathrm{~mm}$.

Estas condiciones climáticas (fig. 2) determinan la existencia de una vegetación clímax representada por sabinares de Juniperus thurifera L. característicos de la asociación Juniperetum phoeniceo-thuriferae (Br.-Bl.y O. Bolós) Rivas-Martínez (Rivas-Martínez, 1978), con diversas subasociaciones relacionadas con cambios microclimáticos vinculados a diferencias de altitud y variaciones en la composición del sustrato (Braun-Blanquet y O. Bolós, 1957).

Siglos de actuación humana en un medio con baja capacidad de respuesta han reducido el primigenio sabinar monegrino a una única muestra ubicada en el paraje conocido como "La Retuerta de Pina" (término municipal de Pina de Ebro, Zaragoza) situado en el cuadrado UTM 30TYL29.

Acotado como dehesa real por el rey aragonés Jaime I (Canellas López, 1988), este bosque quedó al margen de la deforestación brutal del resto de la comarca. permaneciendo prácticamente virgen hasta el año 1906 en que. siendo un monte del Patrimonio del Estado, se privatizó. Si bien desde entonces hasta hoy la regresión del arbolado ha sido muy importante (los mejores suelos están puestos en cultivo y el aprovechamiento de la sabina para leña o como viguería fue muy intenso hasta hace pocos años) quedan todavía 200 Has de sabinar relativamente bien conservado, a pesar de la evidencia de la actuación humana en la

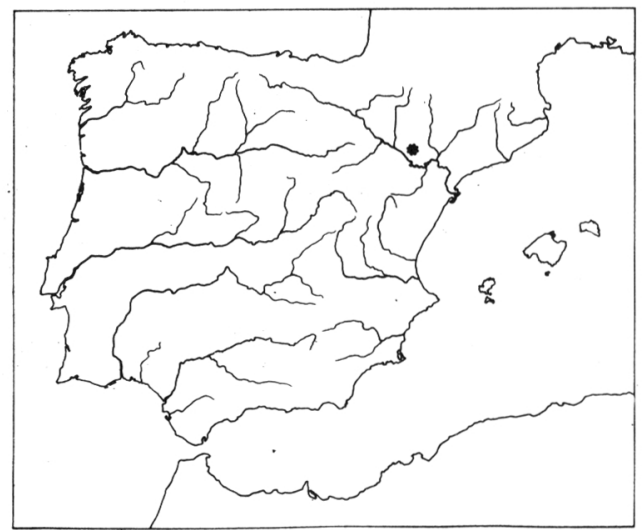

Fig. 1. Localización de la Retuerta de Pina en la Depresión del Ebro, Zaragoza (España).

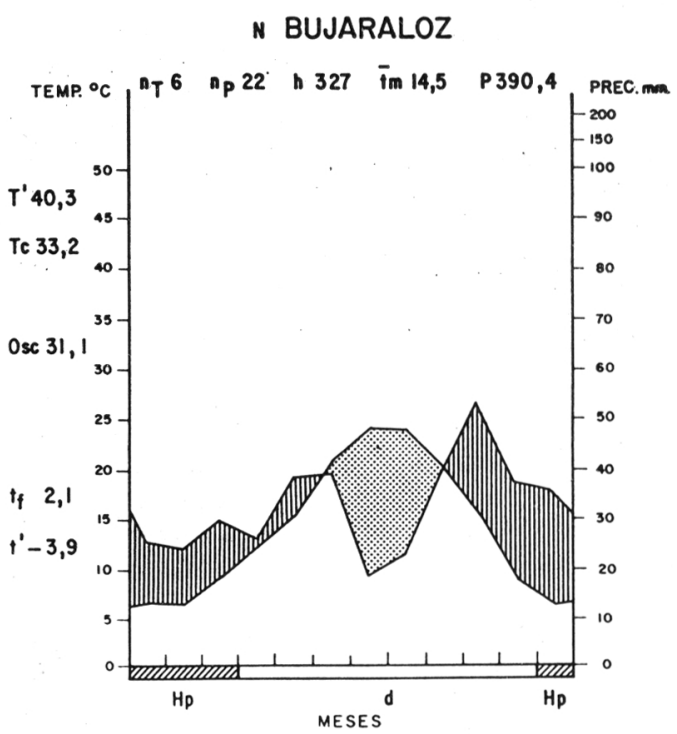

Fig. 2. Diagrama ombroclimático de la estación de Bujaraloz, la más próxima del lugar estudiado (12 $\mathrm{Km})$. 
estructura, constatable en aspectos como la existencia de numerosos ejemplares podados y la escasez de pies vetustos.

Los usos actuales de la zona más relevantes son el cultivo agrícola y la ganadería. Se cultivan cereales de invierno (cebada, trigo y centeno) en secano y con régimen de año y vez. Las condiciones edáficas, la puesta en cultivo de lugares inadecuados y la adversa climatología determinan fuertemente los rendimientos. No obstante, la superficie ocupada por cultivos es muy importante y rompe la continuidad del arbolado.

El aprovechamiento ganadero se limita prácticamente al ganado ovino en régimen extensivo, utilizando los recursos forrajeros de barbechos y rastrojos. También penetra en el sabinar en el que, si la presión es excesiva, impacta fuertemente, debido a la mutilación de los ejemplares y al efecto del pisoteo en el suelo, así como a su enriquecimiento en nutrientes por aporte de heces que hará variar la composición del sustrato arbustivo.

El bosque se asienta en las laderas y cimas de una compleja red de barrancos de fondo plano (llamados vales), cuya facies litológica representativa es la "formación Retuerta" miocénica (Quirantes Puertas, 1978) esencialmente yesífera, a base de yesos masivos blancos o muy claros y limos yesíferos. Alternan con los yesos, margas y arcillas yesíferas.

Estos suelos son poco evolucionados, Entisols del grupo de los Xerorthents (Ministerio de Agricultura, 1978), son pobres y poco profundos, generalmente constituidos por un horizonte A sobre la roca madre. Al ocupar posiciones en pendiente, están sometidos a continua erosión. En el fondo de las vales, se forman suelos algo más evolucionados a base de un horizonte antrópico, sobre un sustrato muy compacto y poco permeable formado por marga yesíferas.

El sabinar corresponde a la subasociación juniperetosum thuriferae Br.-Bl. y O. Bolós. Es la comunidad más pobre en especies de la región y se caracteriza por la presencia de Juniperus thurifera L., Rhamnus lycioides L., Ephedranebrodensis Tineo ex Guss. y Asparagus acutifolius L. El aspecto de esta comunidad es el de un bosque aclarado o estepa arbolada, lo que permite la presencia como sotobosque de diversas comunidades que varían en función de la composición del suelo, la orientación y a altura. Las más destacables son la siguientes:

Lygeo sparti-Stipetum lagascae $\mathrm{Br}$.-Bl. y O. Bolós y Agropyro cristati-Lygeetum sparti Br.-Bl. y O. Bolós o praderas estépicas de gramíneas perennes, que ocupan fondos de vales o la base de laderas orientadas al norte, allí donde se acumulan partículas finas procedentes de la erosión de las laderas. Ononidetum tridentatae Br.Bl. y O. Bolós es un matorral denso y vigoroso, que denota presencia de yesos en profundidad aunque no afloren a la superficie. Ocupa preferentemente laderas suaves y soleadas. La subas. pinetosum halepensis $\mathrm{Br}$.-Bl.y O. Bolós ocupa la parte alta de los cerros, huyendo de la inversión térmica y presentando Pinus halepensis Miller y Juniperus phoenicea L. La asociación Salsolo vermiculatae-Artemisietum albae Br.Bl.y O. Bolós, formada por táxones nitrófilos, aparece como sotobosque del sabinar, en antiguos cultivos colonizados por el arbolado, en el fondo de vales donde se acumula materia orgánica o en lugares muy frecuentados por el ganado.

Una comunidad característica de la degradación avanzada en la serie climácica regresiva de los yesos es el Helianthemetum squamati Br.-Bl. y O. Bolós. Se trata de un matorral aclarado y ralo, que ocupa vertientes erosionadas y secas donde la sabina albar vegeta a duras penas. Cuando la degradación es extrema, el suelo desnudo de las pendientes es ocupado por la subasociación placodietosum $\mathrm{Br}$.-Bl. y O. Bolós cuya mayor biomasa está formada por una costra liquénica, que defiende al suelo de la erosión y que se compone fundamentalmente de: Diploschistes diacapsis (Ach.) Lumbsch ssp. diacapsis, Fulgensia sp. pl., Psora decipiens (Hedw.) Hoffm., Toninia 
sedifolia (Lightf.) Timdal, Acarospora placodiiformis H. Magn., Squamarina sp.pl., Cladonia foliacea (Huds.) Willd., Teloschistes lacunosus (Rupr.) Sav. y otros.

Únicamente señalamos el número de registro con el que están depositados nuestros ejemplares (Herb. Etayo), omitiendo el resto de datos, que son los siguientes: Loc. La Retuerta de Pina, Zaragoza, España. Alt. 330-417 m. UTM 30TYL29, Rec. J. Blasco Zumeta y J. Etayo. Fechas, 6, 7-XII-1989 y 15-III-1991. El herbario particular del primer autor está en disposición de ser consultado en la dirección: Navarro Villoslada 16-3ํㅡㄹ. dcha., 31003-Pamplona, España.

\section{CATÁlogo}

Agonimia cf. octospora Coppins et P. James

Tiene diferencias notables con este taxon, aunque aparentemente el talo es similar a este género, pese a que no forma escuámulas sino gránulos. El córtex, paraplectenquimático, en vez de papilas presenta pelos hialinos, uni- o pluricelulares, de 6-22 $\mu \mathrm{m}$ de largo, visibles incluso a la lupa. Algas verdes, de 8-17 $\mu \mathrm{m}$ de diámetro. Encontrada siempre estéril y a menudo con el talo parasitado por el mixomicete Perichaena corticalis. Rara, Herb. Etayo 5702.

Anaptychia ciliaris (L.) Körb.

Rara.Sólo la encontramos sobre Pinus halepensis Herb. Etayo 5945.

Aspicilia contorta (Hoffm.) Krempelh.

Aproximable a A. lundensis (Fr.) Uloth. que puede ser únicamente un ecotipo lignícola. Esta aparece en regiones montañosas y frías según Clauzade et Roux (1985). Rara, la encontramos en la base de algunos troncos jóvenes en descomposición. Herb. Etayo 5313, 5925.

\section{Athelia arachnoidea (Berk.) Jülich}

Forma manchas orbiculares de color blanco, con cuerpos esclerociales subglobosos de color crema. Parasita a un amplio espectro de líquenes, tanto crustáceos como foliáceos: Diplotomma alboatrum, Candelaria concolor, Hyperphyscia adglutinata, Opegrapha varia, Phaeophyscia insignis, Physcia adscendens, Xanthoria parietina y otros elementos del Xanthorion. Frecuente. Herb. Etayo 5302, 5307, 5928.

Bacidia hegetschweileri (Hepp) Vain.

Apotecios negros pero recubiertos de gran cantidad de pruina blanca y muy convexos cuando maduros. Epitecio negro rojizo, $\mathrm{K}+$ negro violáceo. Hipotecio naranja. Excípulo negro rojizo. Himenio de 40 a $50 \mu \mathrm{m}$. Paráfisis simples, capitadas y recubiertas de una capa irregular gelatinosa, de color rojizo. Ascos octosporados, con las esporas enrolladas en el asco. Esporas de 21-30 x 2-3,5 $\mu \mathrm{m}$, con (3) 5-7 septos y curvadas o vermiformes, la mayoría ensanchadas en uno de los extremos.

Nuestro ejemplar se ajusta bien a otras descripciones de esta rara especie: Sampaio (1921), Clauzade et Roux (1985) y Marcos Laso (1985), excepto en la reacción epitecial con $\mathrm{K}$ (no señalada por estos autores) y, especialmente, en la gruesa cantidad de pruina sobre el disco. Quizás le convenga el rango varietal.

En la segunda cita en territorio español (Marcos Laso,1985). Rara. Herb. Etayo 5929.

\section{Buellia punctata Aggr.}

Talo gris verdoso claro. Apotecios planos, con grueso reborde. Excípulo que se eleva mucho por encima del himenio. Epitecio pardo oscuro e hipotecio pardo. Es notable en esta región la presencia de paráfisis simples, gruesas $(2 \mu \mathrm{m})$, septadas y extraordinariamente capitadas, con ápices que oscilan entre las 4 y $7 \mu \mathrm{m}$ de anchura,pigmentadas interiormente. Himenio de 60 a $70 \mu \mathrm{m}$ y esporas de forma variable, desde suborbiculares hasta reniformes, de $10-13 \times 5,6-8 \mu \mathrm{m}$.

Probablemente, como opinan Nimis y Poelt 91987), conste de varios táxones en la región mediterránea. Local. Herb. Etayo 5946. 
Caloplaca cerina (Ehrht. ex Hedw.) Th. Fr.

Con grandes apotecios pruinosos, similares a los de C. stillicidiorum. La encontramos junto a Rinodina cf. physciospora, Caloplaca citrina, $C$. cf. viperiae y Diplotomma alboatrum. Frecuente. Herb. Etayo 5335, 5336, 5710, 5906, 5916, 5924.

\section{Caloplaca cerina f. ehrartiana}

En la base de las sabinas, aunque bastante rara. Se distingue fácilmente de la anterior por su talo verrucoso-isidioso. Se conoce también en hábitats similares de Navarra (Etayo, 1989). Herb. Etayo 5901.

Caloplaca cerinelloides Poelt.

En ramitas de Salsola vermiculata. Local. Herb. Etayo 5745, 5318.

Caloplaca citrina $(\mathrm{Hoffm}) \mathrm{Th}$. Fr.

Junto a Caloplaca cerinelloides, C. lobulata, Catillaria nigroclavata y otras nitrófilas, tanto en ramitas caídas de Salsola vermiculata L., donde fructifica (5745), como sobre $J$. thurifera. Local, Herb. Etayo 5303, 5306, 5310.

Caloplaca haematites (Chaub. ex St. Am.) Zwackh

Sobre ramitas de Salsola vermiculata. Rara. Herb. Etayo 5745.

Caloplaca lobulata (Flörke) Hellb.

Bien desarrollada en algunos troncos de sabina, junto a Lecanora hagenii, L. chlarotera e Hyperphyscia adglutinata. Local. Herb. Etayo 5309, 5923.

Caloplaca obscurella (Lahm) Th. Fr.

Sólo aparece en la base de gruesos Pinus halepensis. Herb. Etayo 5945.

Caloplaca cf. viperiae Zaahlbr.

Talo indeterminado, blanco, totalmente sorediado bastante grueso; no reacciona con los reactivos habituales. Soredios de 20 a $45 \mu \mathrm{m}$ de diámetro. Ascomas relativamente frecuentes, amarillos, más tarde anaranjados o incluso parduzcos, con reborde de igual color, planos o convexos en la madurez. En los jóvenes, se recubren por un fino reborde talino blanquecino amarillento, de manera similar a $C$. holocarpa. Excípulo propio formado por un prosoplecténquima de luces relativamente gruesas; presenta algunas papilas en el borde. Epitecio y excípulo naranja, $\mathrm{K}+$ púrpura y violeta en la capa externa. Hipotecio compuesto por hifas muy compactadas en paquetes y de gruesa pared, parece cristalífero, pues tiene un intenso color gris al microscopio. Paráfisis septadas, simples o poco ramificadas y de ápice apenas capitado. Esporas de 10-12,5 x 5-7 $\mu \mathrm{m}$, con septo que ocupa la mitad esporal.

Coincide bastante bien con $C$. viperiae Zahlbr. conocida de Dalmacia, excepto en que ésta tiene un talo de color gris verdoso. Bastante frecuente en el sabinar. Herb. Etayo 5655, 5710, 5906, 5915, 5942.

\section{Candelaria concolor (Dicks.) Steiner}

Encontramos talos muy sorediados, acompañando a Hyperphyscia adglutinata, en una sabina muy expuesta. Atacada por Athelia arachnoidea. Rara. Herb. Etayo 5302, 5906.

Candelariella aurella (Hoffm.) Zahlbr.

Frecuente en ramitas caídas de romero y sobre madera de sabina cubierta de cianobacterias. Herb. Etayo 5273, 5463.

Candelariella xanthostigma (Ach.) Lett.

Local en troncos de sabina. Herb. Etayo 5906, 5915.

Catillaria nigroclavata (Nyl.) Schuler

Muy variable, encontramos algunos talos casi isidiados, con abundantes picnidios inmersos y conidios de $2-3 \times 0,3 \mu \mathrm{m}(5308,5306)$. Otros talos tienen apotecios de gran tamaño, que alcanzan los $0,5 \mathrm{~mm}$ de diámetro $(5322,5741)$. Sobre ramitas caídas de romero, sabina y Salsola. Común. Herb. Etayo 5745, 5305, 5912,5914, 5941. 
Chrysothrix candelaris (L.) Laundon

En una vieja sabina, junto a Lecanora horiza, Hyperphyscis adglutinata y Xanthoria parietina. Rara. Herb. Etayo 5317, 5922.

Diplotomma alboatrum (Hoffm.) Flotow

Muy abundante, tanto en corteza como sobre madera de sabina. Herb. Etayo 5327, 5336, 5712, 5906, 5920 .

Endocarpon pusillum Hedw.

Especie terrícola, que puede colonizar la base de las sabinas en las orientaciones más húmedas. Esporas de 34-37 x 13-21 $\mu \mathrm{m}$ y de color rosado y con algas himeniales redondas de 3-5 $\mu \mathrm{m}$. Común sobre suelos, rara como epifita. Herb. Etayo 5316.

Evernia prunastri (L.) Ach.

Sólo aparece en Pinus halepensis y Cistus clusii Dunal. Rara. Herb. Etayo 5912.

Hyperphyscia adglutinata (Flörke) Mayrh. Poelt.

A veces forma masas totalmente sorediadas. Raramente fructificada $(5324,5325)$, sobre madera de sabina. En ocasiones cubierta por Licea kleistobolus. Muy abundante. Herb. Etayo 5309, 5310, 5314, 5315, 5317, 5322, 5703, 5906, 5926.

Hypogymnia physodes (L.) Nyl.

Sobre Pinus halepensis en las situaciones más húmedas. Herb. Etayo 5947.

Lecania viridulo-granulosa (Harm.) Zahlbr.

Apotecios típicos de este taxon, con un grueso córtex de 40 a $50 \mu \mathrm{m}$, disco muy pruinoso y esporas de $11-15 \times 3-5 \mu \mathrm{m}$, tanto con 1 como con 3 septos. Sin embargo, el talo es diferente a otros vistos por nosotros (Navarra, Etayo, 1989), pues consta de gránulos erumpentes formando soralios, que en muchas partes forman un talo pulverulento, de color verde claro. Rara. Herb. Etayo 5935.

Lecanora carpinea (L.) Vain.

Sobre arbustos rastreros. Local. Herb. Etayo 5948.

Lecanora chlarotera Nyl.

Sobre sabina y ramas rastreras de romero. Algunos ejemplares pueden adscribirse a $L$. rugosella Zahlbr. Común Herb. Etayo 5309, 5332.

Lecanora expallens Ach. 5939.

Estéril, como es habitual en esta especie. Probablemente inframuestreada. Herb. Etayo

Lecanora hagenii (Ach.) Ach.

Variable, en las partes más ocultas y protegidas forma talos pulverulentos. Muy abundante. Herb. Etayo 5309, 5312, 5327, 5336, 5455, 5273, 5915.

Leconora horiza (Ach.) Linds.

Muy común en todo tipo de substratos. Herb. Etayo 5301. 5317, 5310, 5320, 5712, 5906, 5915, 5938.

Lecidella achristotera (Nyl.) Hertel et Leuckert

Sobre sabina decorticada. La base del himenio e hipotecio son especialmente inspersas. Frecuente. Herb. Etayo 5323, 5912, 5934.

Lecidella elaeochroma (Ach.) Choisy

Frecuente sobre sabina y romero. Herb. Etayo 5223, 5332, 5933. 
Lepraria crassissima (Hue) Lett. Etayo 5921.

Habitualmente terrícola. A menudo coloniza la base y tocones de las sabinas. Herb.

\section{Licea kleistobolus G.W. Martin}

Tanto sobre corteza como sobre líquenes corticícolas, especialmente Hyperphyscia adglutinata, en viejas sabinas. No aparece citado sobre líquenes en Clauzade, Diederich y Roux (1989). Local. Herb. Etayo 5703, 5747.

Licea parasitica (Zukal) G.W. Martin

Mixomicete frecuente sobre diversas especies de líquenes (Etayo, 1989), en esta localidad lo hace especialmente sobre Hyperphyscia adglutinata y Xanthoria parietina. Local. Herb. Etayo 5311, 5315.

Melaspilea proximella ( $\mathrm{Nyl}$.) Nyl.

Aparece en fisuras, formando grupos compactos de varios individuos, pero también de manera solitaria. Local. Herb. Etayo 5915.

Mycocalicium parietinum (Ach. ex Schaer.) Hawksw.

Muy rara, sobre madera vieja y dura de sabina. Herb. Etayo 5609.

Opegrapha varia s.l.

Aparece muy frecuentemente en las bases sombreadas de gruesas sabinas copudas. En todos los casos, los ascomas son de disco corto, muy abierto y sobresaliente del sustrato y suelo estar acompañada por Diplotomma alboatrum. Herb. Etayo 5331, 5336, 5906, 5932.

Orbilia coccinella (Sommerf.) Karst.

Fundamentalmente lignícola, aparece también sobre otros líquenes corticícolas y algas verdes. Rara. Herb. Etayo 5326.

Pachyphiale arbuti (Bagl.) Arnold

Muy rara y con muy escasos apotecios, siempre protegido entre otros líquenes de mayor desarrollo como Hyperphyscia adglutinata o Rinodina cf. physciospora. Herb. Etayo 5304.

Parmelia glabratula (Lamy) Nyl.

Sobre arbustos rastreros. Rara. Herb. Etayo 5949.

Perichaena corticalis (Batsch) Rostaf.

Lignícola y ocasionalmente sobre líquenes epifitos. No aparece reseñada en Clauzade, Diederich y Roux (1989). Rara. Herb. Etayo 5749.

Pertusaria paramerae Crespo et Vèzda

Ya citada de esta localidad por Crespo y Vèzda (1985). Aparece únicamente en la base de algunas sabinas sombreadas o raíces decorticadas (5328), formando grandes talos. La muestra 5654 está parasitada por Sphinctrina turbinata. Local. Herb. Etayo 5319, 5326, 5330, $5907,5927$.

Phaeophyscia cernohorskyi (Nádv.) Essl.

Frecuente junto a Catillaria nigroclavata, Hyperphyscia adglutinata y Caloplaca $\mathrm{cf}$. viperiae. Herb. Etayo 5306, 5314, 5605.

Phaeophyscia insignis (Mereschk.) Moberg

Pasa desapercibida por su pequeño tamaño, aunque probablemente no sea muy rara sobre ritidoma de J. thurifera. Herb. Etayo 5951

Phaeophyscia nigricans (Flörke) Moberg

En comunidades del Xanthorion parietinae. Local. Herb. Etayo 5334, 5931. 
Phaeophyscia orbicularis (Neck.) Moberg

Frecuente. Herb. Etayo 5906, 5940.

Physcia adscendens (Fr.) Oliv.

Frecuente. Herb. Etayo 5906, 5916, 5937.

Physcia biziana (Massal.) Zahlbr.

Frecuente. En el norte peninsular se encuentra desde áreas subáridas hasta subhúmedas.

Herb. Etayo 5952.

Physcia biziana var. leptophylla VÈzda

Más rara que la variedad típica. Herb. Etayo 5330, 5930.

Physcia tenella (Scop.) DC.

Local. Herb. Etayo 5316.

Physconia perisidiosa (Erichs.) Moberg

Especie nitrófila que se encuentra, raramente, colonizando amplias superficies en la base de las sabinas. 5953 .

Pseudevernia furfuracea (L.) Zopf

Sobre Pinus halepensis, no aparece en las sabinas. Herb. Etayo 5954.

Pyrenochaeta xanthoriae Diederich (fig. 3)

Celomicete parásito. Picnidios diminutos de $0,2 \mathrm{~mm}$ de alto y $0,15 \mathrm{~mm}$ de ancho, de color negro, cubiertos de pelos en su parte superior. Estos son pardos, septados y simples, de 70-110 × $3 \mu \mathrm{m}$. Pared del picnidio paraplectenquimática, de color gris. Conidios acropleurógenos, que salen en cadenas celulares, de aproximadamente $25 \times 2,5 \mu \mathrm{m}$. Conidios desde elipsoidales a subesféricos, de 3-4 × $2 \mu \mathrm{m}$, hialinos.

Este parásito, recientemente descrito (Diederich, 1990) nos aparece sobre el talo de Xanthoria parietina, especialmente sobre aquellas partes negras, colonizadas por Xanthoriicola physciae. Especie sólo conocida hasta el momento de Luxemburgo. Rara. Herb. Etayo 5955. Rinodina cf. physciospora (Choisy et Werner) Zahlbr.

Talo pesudoisidiado, irregular, corticado, con córtex paraplectenquimático de 20 a 30 $\mu \mathrm{m}$ de espesor, con abundantes apotecios planos, pardos o negros, de 0,3 a $0,6 \mathrm{~mm}$, de borde bien visible, a veces oculto por un desarrollo fuerte del excípulo prosoplectenquimático e I-, que parece transformarse en paraplectenquimático con el tiempo. Epitecio pardo, con un pigmento pardo, $\mathrm{P}-$, en el interior de las paráfisis. Ascos octosporados pero con muchas esporas abortadas (suelen aparecer de 1 a 4 esporas en buen estado por asco). Esporas de 14-17 x 6$8 \mu \mathrm{m}$ de tipo genarii. Por este carácter se relaciona con $R$. genarii pero su talo es mucho más desarrollado (Mayrhofer com. pers.).
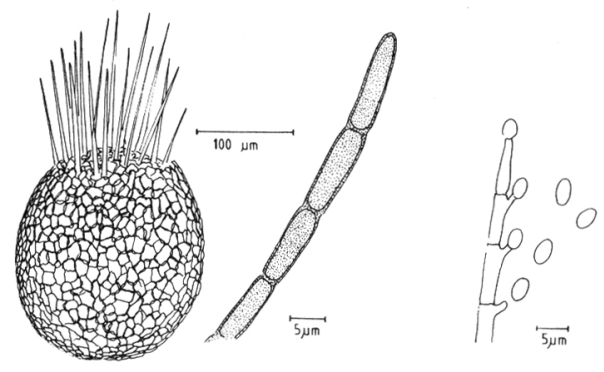

Fig. 3. Pyrenochaeta xanthoriae: picnidio con pelos apicales. Detalle de un pelo. Conidios acropleurógenos. 
R. physciospora puede coincidir bien con esta especie en cuanto a las esporas (tamaño) y hábitat (Marruecos, sobre Abies, si bien a elevada altitud: $1650 \mathrm{~m}$ ). No hemos podido obtener el material de los Herbarios donde se encontraba.

Bastante frecuente. Herb. Etayo 5310, 5312, 5321, 5929.

\section{Sclerophora sp.}

Diminuto calicial de color negro, con macedio naranja pardo. Estipe de 0,15-0,2 mm de largo y $0,05 \mu \mathrm{m}$ de espesor. Cúpula del ascoma semiesférica, de $0,2 \mathrm{~mm}$ de alta y $0,25 \mathrm{~mm}$ de diámetro como máximo. Pared de color pardo rojizo, $\mathrm{K}+$ verde azulado intenso. Ascos biseriados, con esporas orbiculares de 3-4 $\mu \mathrm{m}$, lisas e incoloras. Según Tibell (com. Pers.), se trata de un taxon no descrito, anteriormente conocido de Italia, probablemente perteneciente a éste género, pese a tener el excípulo y estipe formado por hifas oscuras.

Lo más curioso es su hábitat, pues la mayoría de las veces, se encuentra sólo en la parte interna, nada expuesta, del ritidoma que se va descamando. Muy disperso y muestreado al azar. Herb. Etayo 5703, 5736.

\section{Sphinctrina turbinata (Pers. ex Fr.) De Not.}

De carácter suboceánico según Lofgren y Tibell (1979), sobrevive también en climas secos y semiáridos. Sus esporas orbiculares de 8-15 $\mu \mathrm{m}$ y la falta de reacción del excípulo con el $\mathrm{K}$ la acercan a este taxon, pese a que no presenta la característica capa hialina externa recubriendo el excípulo y pie de S. turbinata.

Rara sobre Pertusaria paramerae. Herb. Etayo 5654.

Taeniolella phaeophysciae D. Hawksw.

Sobre Hyperphyscia adglutinata, junto con Athelia archnoidea. Herb. Etayo 5307.

Teloschistes lacunosus (Rupr.) Sav.

Considerado como terrícola. Sin embargo, parece que el comienzo de su desarrollo lo efectúa, siempre, sobre ramas, tanto de sabina como otros arbustos, en posiciones muy cercanas al suelo. Frecuente. Herb. Etayo 5902, 5910.

Thelenella justii (Serv.) Mayrh. et Poelt.

Talo granuloso bien desarrollado, casi pseudoisidiado, con algas verdes. Peritecios inmersos, apenas visibles, de pared hialina, muy gruesa, diferenciada en dos capas, una externa prosoplectenquimática y otra interna finamente paraplectenquimática. Sin perifisoides. Parafisoides ramificadas y ascos bitunicados con 2 esporas maduras y el resto abortadas, que suelen permanecer en la parte apical. Asco con engrosamiento apical I-, donde no se forma una cámara apical, aunque sí se observan tres diminutas elevaciones, similares a las del género Protothelenella (Mayrhofer y Poelt, 1985). Esporas murales, de forma variada, de 27-46 x 12$18 \mu \mathrm{m}$. Picnidios de similar aspecto a los peritecios, de hasta $0,3 \mathrm{~mm}$, de pared prosoplectenquimática, cuya parte más interna está coloreada de pardo. Conidióforos cilíndricos o piriformes de 5-7 x $1 \mu \mathrm{m}$ y conidios curvados de 11-17 x 0,5 $\mu \mathrm{m}$.

Muy rara, se encuentra en zonas húmedas de la corteza, acompañada por Orthotrichum diaphanum Brid. y Agonimia cf. octospora.

Especie poco muestreada, únicamente se conoce de Grecia (localidad tipo), sobre Abies cephalonica, Francia (Bricaud y Roux, in Van Haluwyn y Letrouit-Galinou, 1990) y de Argelia sobre Pinus halepensis (Van Haluwyn y Letrouit-Galinou, 1990). Tercera cita europea. Herb. Etayo 5956.

Trichothecium roseum (Pers. ex Fr.) Link (fig. 4)

Sobre Lecanora horiza en una rama cercana al suelo, recubriéndola por completo y con aspecto de filamentos erectos capitados. Según W. Gams (com. pers.) es un micoparásito destructivo, muy común sobre todo tipo de sustratos fúngicos y se diferencia de otros géneros similares en aspecto, como Arthrobotrys por sus conidióforos no ramificados y conidiogénesis retrogresiva.

En pocas ocasiones citada sobre líquenes. Herb. Etayo 5754. 
Usnea hirta (L.) Web. ex Wigg.

No coloniza las sabinas, pero sí las ramas de de Cistus clusii, en las zonas más húmedas. Herb. Etayo 5957.

\section{Verrucaria sp.}

En estudio, junto a otros ejemplares del género, de hábitat corticícola, de Navarra. Rara. Herb. Etayo 5958.

Vouaxiella verrucosa (Vouaux) Petr. et Syd. Se distingue de $V$. lichenicola por sus conidios verrucosos, oscuros, de 9-14 x 4,5-5 $\mu \mathrm{m}$. La encontramos siempre sobre su huésped típico Lecanora horiza. Conocida de las Islas Británicas, Pakistán (Hawksworth, 1981), Argelia (Van Halluwyn y Letrouit-Galinou, 1990) y España (Giralt, 1991). Frecuente en la zona. Herb. Etayo 5310, 5328, 5697, 5943.

Xanthoria parietina (L.) Th. Fr.

Muy común en el sabinar. Herb. Etayo 5315, 5317, 5325, 5916, 5919.

Xanthoriicola physciae (Kalchbr.) Hawksw.

Junto a Pyrenochaeta xanthoriae es un parásito frecuente de Xanthoria parietina. Herb. Etayo 5959.

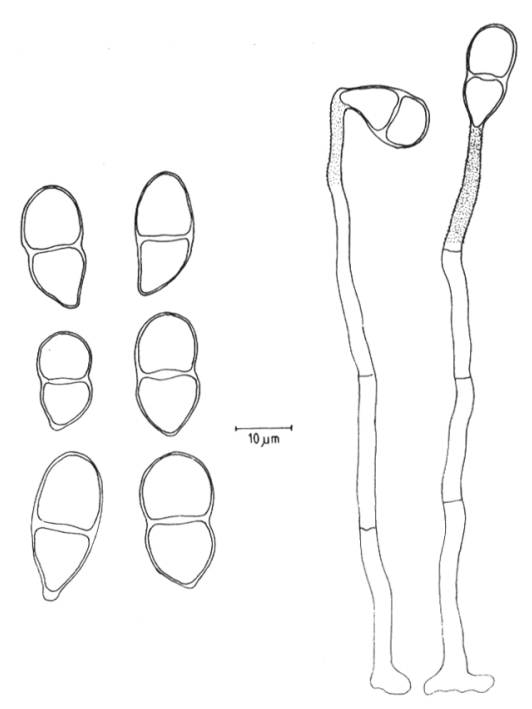

Fig. 4. Trichothecium roseum: conidios septados y conidióforos hialinos.

\section{CONCLUSIONES}

Se han muestreado un total de 66 táxones en el sabinar de La Retuerta de Pina, enclavado en Los Monegros (Zaragoza, España). A pesar de la extrema sequedad y continentalidad climática de esta parte de la Depresión del Ebro, el recubrimiento liquénico de las sabinas es muy notable y presenta una flora peculiar. Esta originalidad también se da en otros grupos vegetales y animales propios de esta hábitat. Un hecho preocupante es la extrema deforestación llevada a cabo sobre estos notables bosques en esta parte de España, actualmente relegados a puntos residuales.

La flora liquénica está compuesta por una mezcla de especies xéricas banales consideradas como más o menos nitrófilas (Xanthoria parietina, Physcia s.l., Diplotomma alboatrum, Caloplaca spp., Lecidella spp., etc.) y otras, mucho más escasas, igualmente adaptadas a ambientes muy secos, de óptimo en bosques de tipo continental, como Pertusaria paramerae o Thelenella justii.

El recubrimiento de los troncos es, fundamentalmente, a base de especies crustáceas o pequeñas foliáceas (Physcia, Phaeophyscia o Physconia) no apareciendo táxones de talo más desarrollado, excepción hecha de Teloschistes lacunosus, especie adaptada a estas condiciones. Otras más mesófilas de talos foliáceos o fruticulosos (Parmelia glabratula, Usnea hirta), se ven relegadas a las partes más húmedas del bosque o bien a las ramas inferiores del estrato arbustivo, que por su cercanía al suelo son las más húmedas. Sclerophora sp., representante de un orden sin representantes xerófilos, adopta una curiosa estrategia, como la de desarrollarse en la parte interna 
del ritidoma. La ausencia de luminosidad de estos ambientes, ha condicionado, probablemente, su desarrollo fúngico.

Parece interesante profundizar en el futuro en el hecho de que muchos táxones, en estos ambientes presentan talos más desarrollados, en muchos casos sorediados o pseudoisidiados, que los típicos. Esto sucede por ejemplo con: Catillaria nigroclavata, Lecanora hagenii, Lecania viridulo-granulosa, etc. En algunas partes del tronco la mezcla de éstas y otras menos conocidas como: Caloplaca aff. viperae o Rinodina cf. physciospora, produce masas donde es imposible distinguir o separar los talos de una u otra especie.

Por último se han recolectado 11 especies de hongos liquenícolas, lo que supone un factor indicativo de la estabilidad del bosque. Algunos no habían sido señalados en la Península y sorprenden las tres especies de mixomicetes encontradas, de las cuales de dos no conocíamos citas anteriores sobre líquenes.

AGRADECIMIENTOS. Agradecemos a los doctores P. Diederich (Luxemburgo), W. Gams (Baarn), E. Gracia (Barcelona), H. Mayrhofer (Graz), J. Poelt (Graz) y L. Tibell (Uppsala) su ayuda con algunos táxones conflictivos. El primer firmante también debe agradecer la concesión de una beca adscrita al proyecto de investigación PB89-0518-C02-01 financiado por la D.G.I.C.Y.T.

\section{BIBLIOGRAFÍA}

BRAUN-BLANQUET, J \& O. BOLÓS -1957- Les groupements végétaux du basin moyen de l'Ebre et leur dynamisme. An. Est. Exper. Aula Dei, 5(1-4), 266 pp., Zaragoza.

CANELlAS LÓPEZ, A. -1988-Diplomatario medieval de la Casa de Ganaderos de Zaragoza. Real Soc. Arag. de Amigos del País, 533 pp, Zaragoza.

CLAUZADE, G., P. DIEDERICH \& C. ROUX -1989- Nelikengintaj fungoj. Ilustrita determinlibro. Bull. Soc. Linn. Prov. num. spéc., 1: 1-142.

CLAUZADE, G. \& C. ROUX -1985- Likenoj de Okcidenta Europo. Ilustrita determinlibro. Bull. Soc. Bot. Centre-Ouest, num. spéc., 7: 1-893.

CRESPO, A. y A. VĖZDA -1985- Pertusaria paramerae sp. nov., un liquen epifito de los sabinares españolas. Anales J. Bot. Madrid, 41(2): 251-255.

DIEDERICH, P. - 1990- New or interesting lichenicolous fungi. I. Species from Luxembourg. Mycotaxon, 38: 297-330.

ETAYO, J. -1989- Líquenes epifitos del Norte de Navarra. Tesis Doctoral ined., Universidad de Navarra, $980 \mathrm{pp}$.

GIRALT, M. -1991- Flora $i$ vegetacio liquenica epifitica de la plana i serralades litorals tarragonines. Tesis Doctoral ined., Universidad de Barcelona, 574 pp.

HAWKSWORTH, D.L. -1981- The lichenicolous Coelomycetes. Bull. Br. Mus. Nat. Hist. (Bot.), 9: 1-98.

LÖGFREN, O. \& L. TIBELL -1979- Sphinctrina in Europe. Lichenologist, 11: 109-137.

MARCOS LASO, B. -1985- Flora y vegetación liquénica epifítica de las sierras meridionales salmantinas. Serie resúmenes. Tesis Doctorales. Ed. Univ. Salamanca.

MINISTERIO DE AGRICULTURA -1978- Mapa de cultivos y aprovechamiento E: 1-:50000, hoja 413-GELSA. Subdirección general de producción vegetal, $38 \mathrm{pp}$ y plano. Madrid.

MINISTERIO DE AGRICULTURA, PESCA Y ALIMENTACIÓN -1987- Caracterización agroclimática de la provincia de Zaragoza. Dirección gral. de Producción Agraria. Madrid, $195 \mathrm{pp}$.

MAYRHOFER, H. \& J. POELT -1985- Die Flechtengattung Microglaena sensu Zahlbruckner in Europa. Herzogia, 7: 13-79.

NIMIS, P.L. \& J. POELT -1987- The lichens and lichenicolous fungi of Sardinia (Italy), an annotated list. Studia Geobot., 7, suppl. 1: 1-269.

OCHOA JARAUTA, M.J. -1982-Relaciones entre el medio y comunidades vegetales del sabinar continental árido en el Valle del Ebro. INIA, $52 \mathrm{pp}$, Madrid. 
QUIRANTES PUERTAS, J. -1978- Estudio sedimentológico y estratigráfico del terciario continental de los Monegros. C.S.I.C., 200 pp., Zaragoza.

RIVAS-MARTÍNEZ, S. -1987-Memoria del mapa de series de vegetación de España. ICONA, $268 \mathrm{pp}$, Madrid.

SAMPAIO, G. -1921- Novas contribuiçoes para o estudo dos líquenes portugueses. Brotéria, Série Botânica, 19(1): 12-36.

VAN HALLUWYN, C. \& M.A. LETROUIT-GALINOU -1990- La flore lichénique de Pinus halepensis dans la région de Tebessa (Algérie Oriental). Cryptogamie, Bryol. Lichénol. 11(1): 31-42.

(Aceptado para su publicación en mayo de 1992)

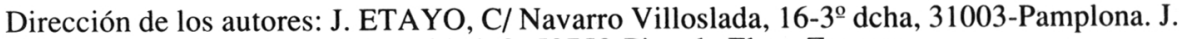
BLASCO-ZUMETA, C/ Hispanidad, 8. 50750-Pina de Ebro, Zaragoza. 